This article has been scanned by iThenticat No plagiarism detected

Volume 3, Issue 4, August 2021

p. 382-389

\title{
RECRUIT SPATIAL SYMBOLS IN BRAND DESIGN
}

http://dx.doi.org/10.47832/2757-5403.4-3.33

Dina Mohammed INAD 1

\begin{abstract}
:
Brand plays a major role in the success of companies, the corporate identity has become firmly established in the business communication environment, and can play a major role in the success of the company. Spatial symbols do not take a mere narrative structural formation, because their presence is not a neutral, static presence, but rather plays an active role in brand design and even in determining its functional options.

Accordingly, dealing with these symbols was of great importance, as they are one of the components of the semantic construction of the design work, It is one of the foundations that constitute the designer's vision towards achieving the desired goal of the design, In this context, the search for spatial symbols becomes justified, especially if the design is among the works that show the act of belonging to the place.

the importance of the tagged study (the employment of spatial symbols in the design of brands) to clarify the subject through three chapters. The first chapter includes the research problem and the need for it Which is represented by the following question (What are spatial symbols and how are they employed in the brand)? The aim of the study was (to identify spatial symbols in brand design). It includes the importance of the research, the limits of the research, and the definition of terms.

As for the second chapter, it included the theoretical framework consisting of four sections that included the first topic (the symbol and identity of the place) and the second topic (symbols in graphic design), while the third topic included (the brand design). The fourth topic included (research applications), as the researcher adopted the descriptive approach, the method of content analysis in analyzing research models.

In the third chapter, the research reached the results and conclusions, including:

1-As a result, the spatial symbols in the brand achieve the depth of belonging and the cultural embodiment of the country to be employed in the brand.
\end{abstract}

\footnotetext{
${ }^{1}$ Dr. , Baghdad University, Iraq, Kamrdi@yahoo.com
} 
2-The spatial symbols in the design of the brand have had a creative impact on the recipient, and it helps him identify the spatial references of the industrial establishment (cement factories).

Key words: Spatial Symbols, Design, Brand.

\title{
توظيف الرموز المكانية في تصميم العلامات التجارية
}

\author{
2
}

\begin{abstract}
الملخص

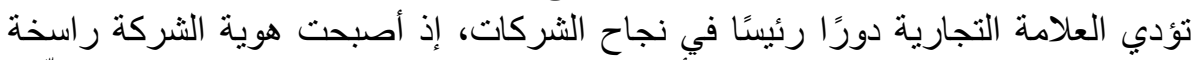

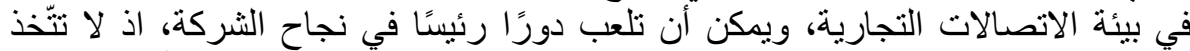

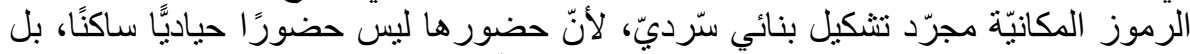

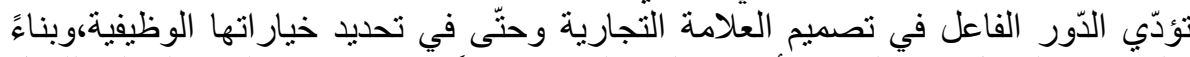

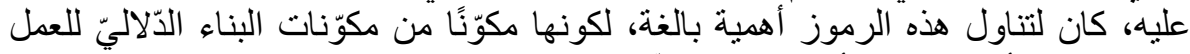

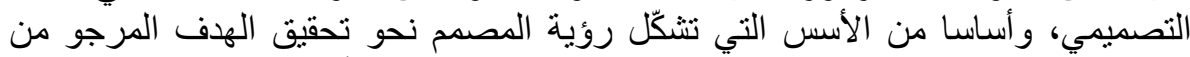

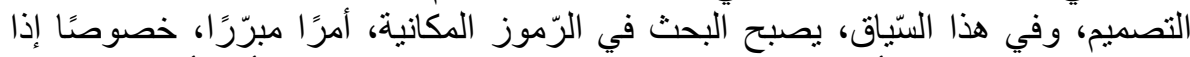

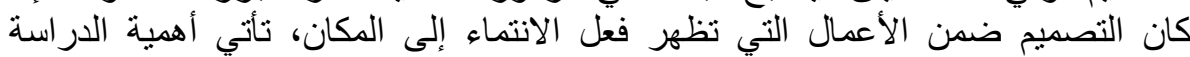

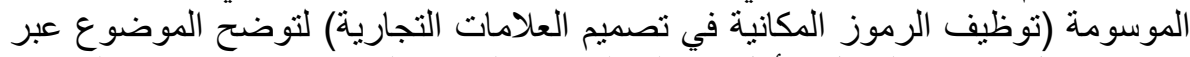

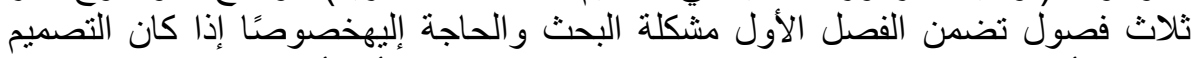

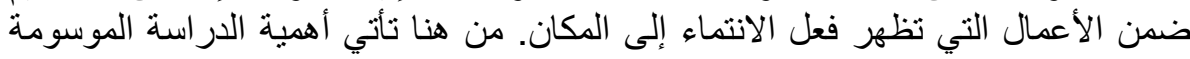

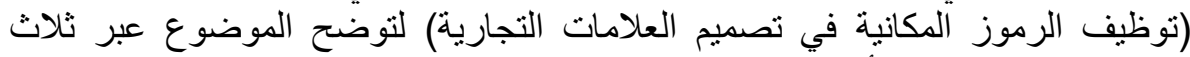

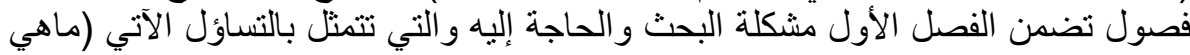

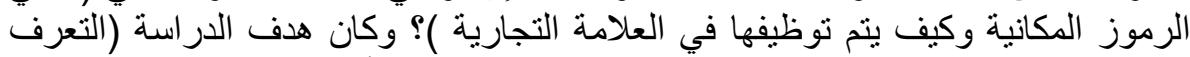
على الرموز المكانية في تصميم العلامة التجارية). وتضمن أهمية البحث الهث وحدود البحث

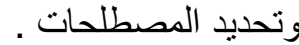
أما الفصل الثاني تضمن الاطار النظري مكون من أربع مباحث تضمن المبحث الأول المبرل

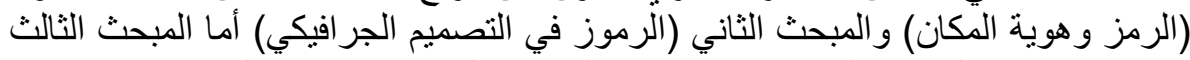

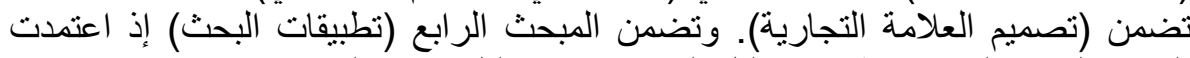

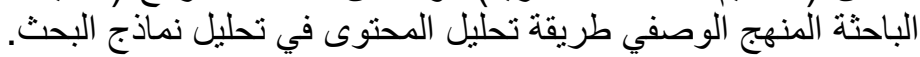

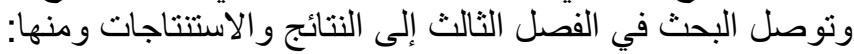

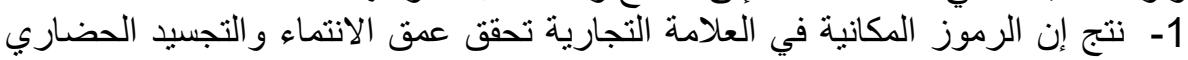
للبلد ليتم توظيفها في العلامة التمانة التجارية .

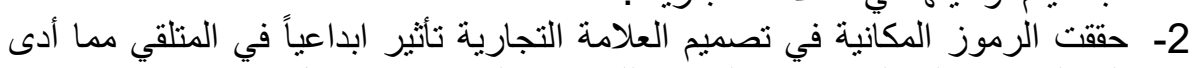

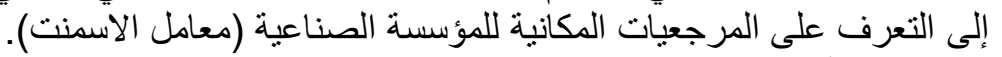
الكلمات المفتاحية: الرموز المكانية، التصميم، العلامة التجارية.
\end{abstract}

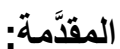

يتحدد الاهتمام المتز ايد بمشكلة تصميم و إدارة العلامات التجارية عن طريق فيق حقيقة أن العلامات التجارية المعروفة هي

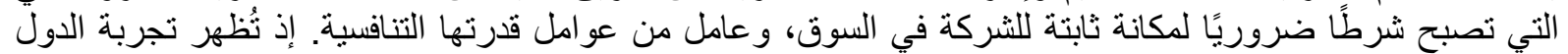

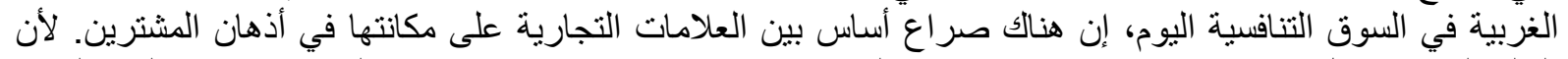

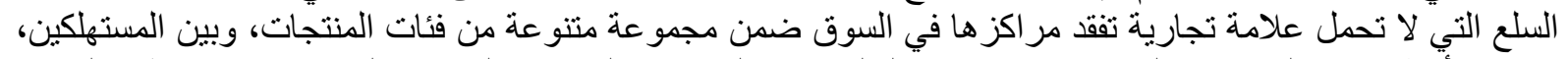

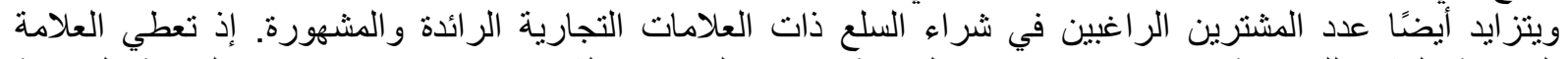

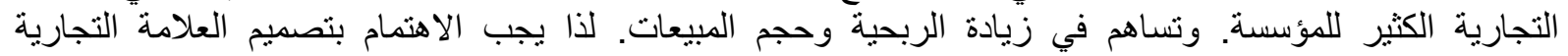


وتضمينها العناصر والرموز المكانية ذات الفاعلية على التأثير والاقناع للمستهلك تحقيقاً للوظيفة التصميمية، وتجسيداً للقيم الحضارية و البيئية لمكان المنتج للسلعة.

$$
\begin{aligned}
& \text { الفصلة الأول } \\
& \text { مشكلة البحث والحاجة إليه: } \\
& \text { يمكن تحديد مشكلة البحثث بالتساؤل الآتي (ماهي الرموز المكانية وكيف يتم توظيفها في العلامة التجارية)؟ }
\end{aligned}
$$

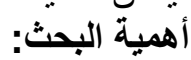

$$
\begin{aligned}
& \text { تكمن أهمية البحث في انه: }
\end{aligned}
$$
الوظُيفة: (هي الفائدة المعنية التي يحققها الثيء).(هيغل، 1986، 1976، 586) وهي (العمل الخاص الذي يقوم به الفرد في

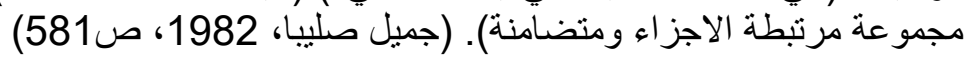

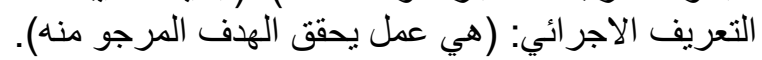

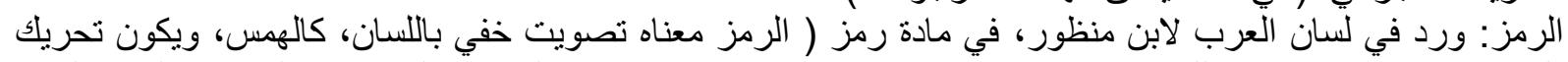

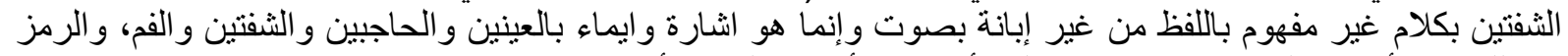

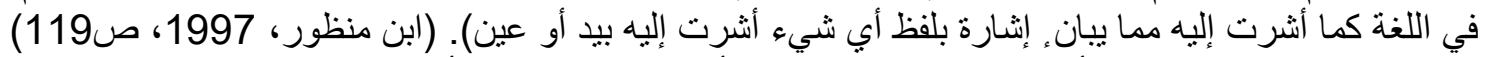

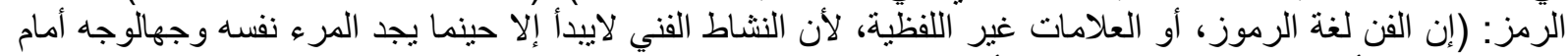

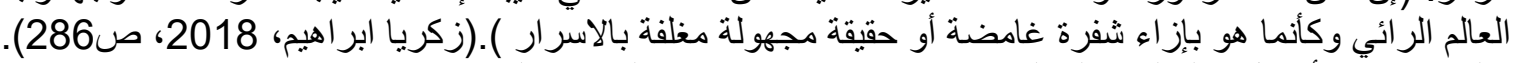

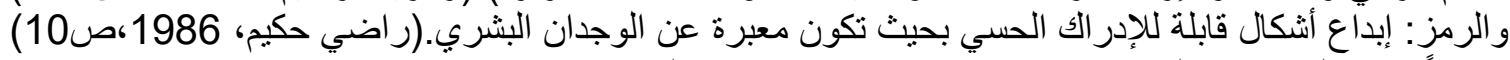

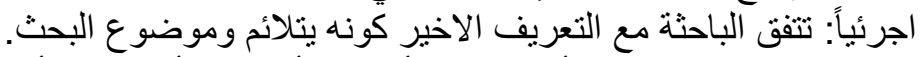

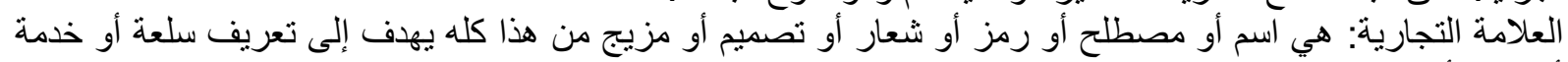

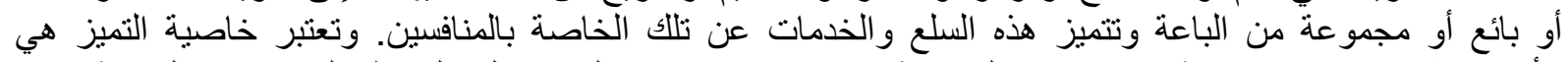

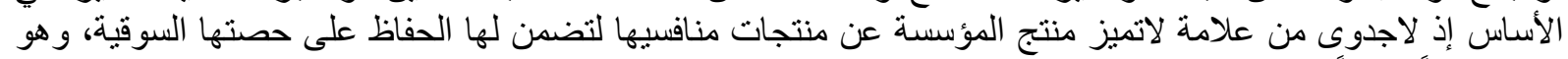

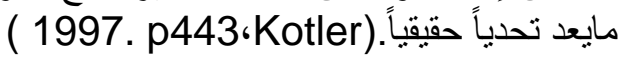

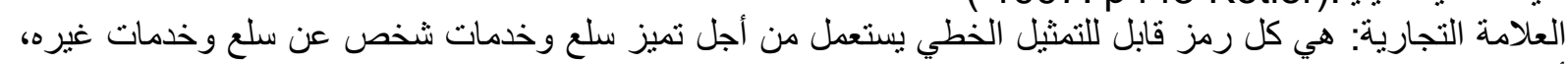

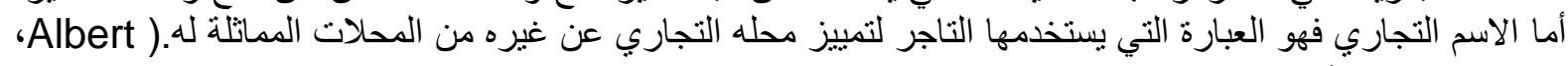

( p 450،1998 اجرئياً: تتفق الباحثة مع التعريف الثناني كونه يتلائم وموضوع البحث.

\section{المبحث الأول

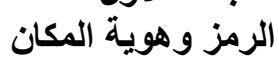

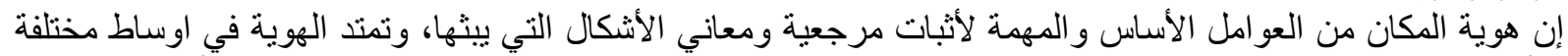

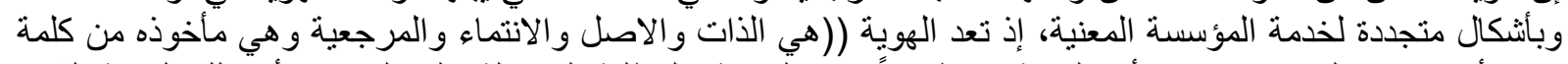

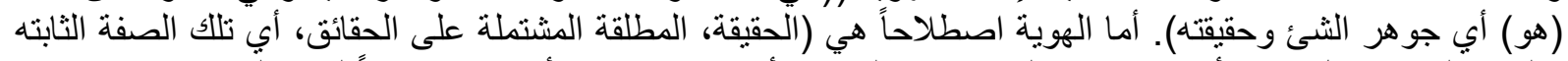

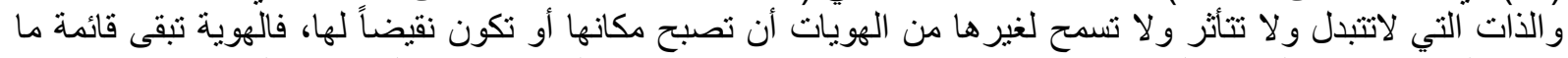

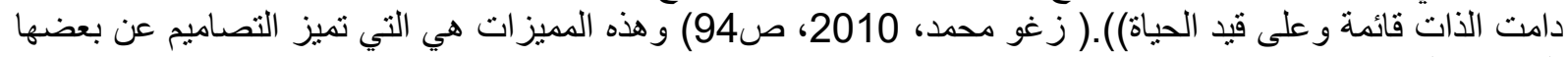
البعض والتي تعبر عن شخصيتها و وحضارتها وورجودها.

${ }^{*}$ https://icsc.gov.iq/ 


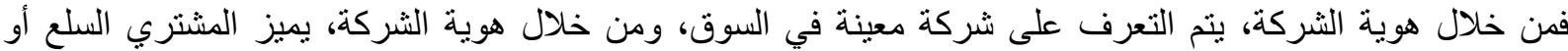

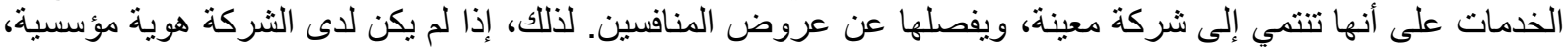

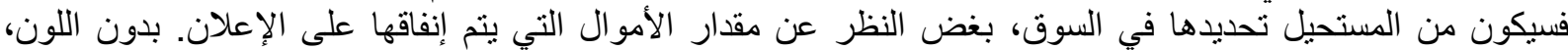

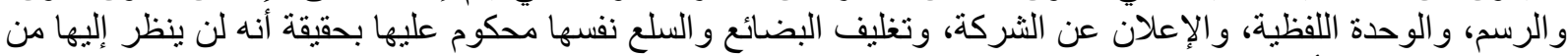

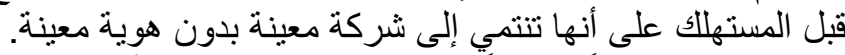

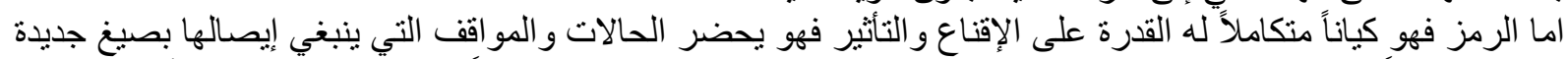

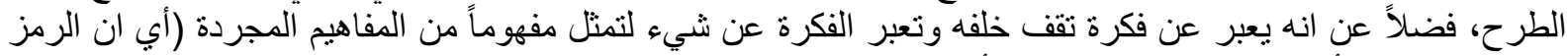

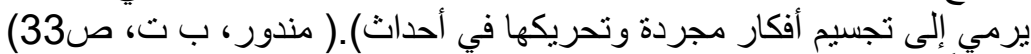

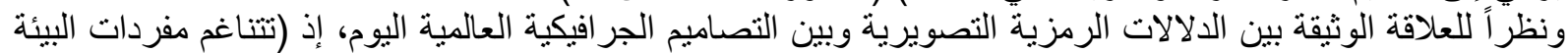

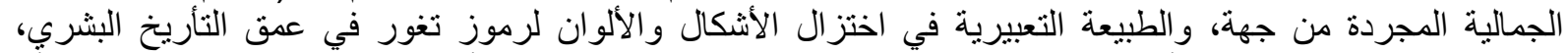

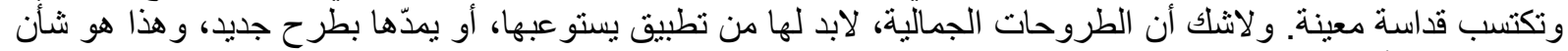

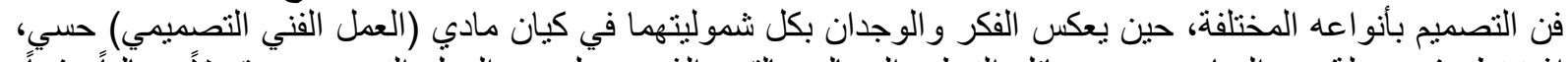

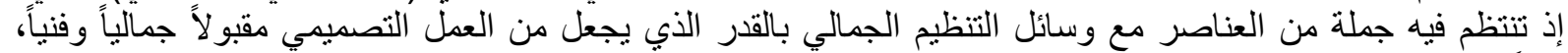

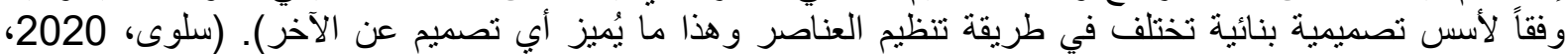

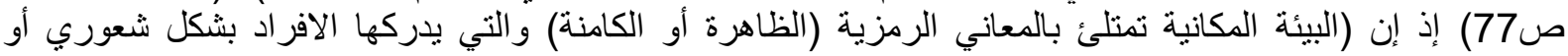

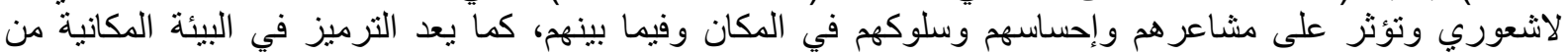

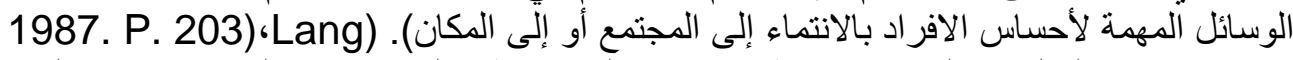

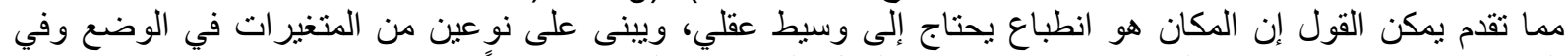

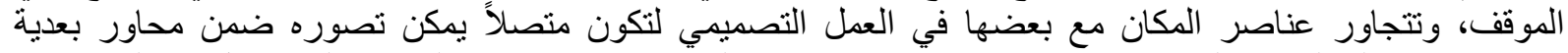

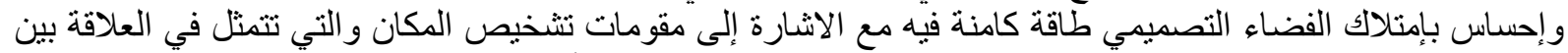

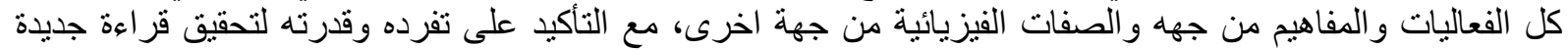

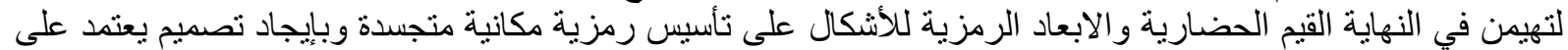
مرجعية حقيقية ثابتة.

\section{الرموز في التصميم الجرافيكي}

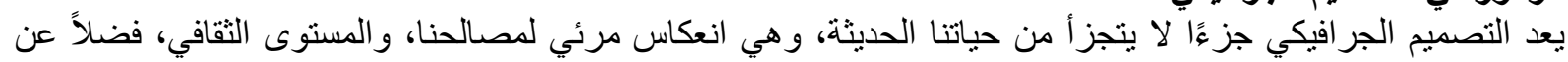

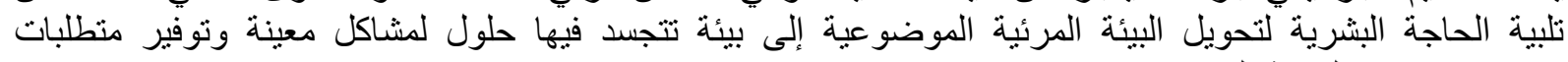
واحتياجات سايكولوجية الفرد.

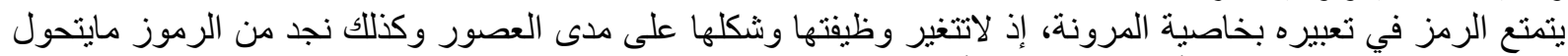

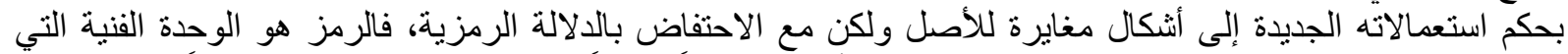

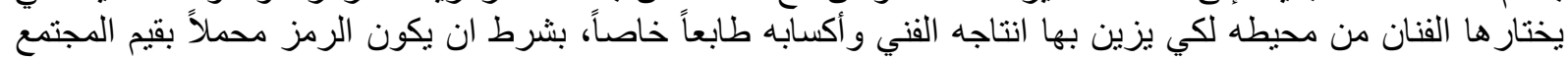
الثقافية. فالرمز هو ( العلامة الحقيقة أو العنصر الحقيقي الذي يتم من خلاله معرفة الكثير من تاريخ أو تراث شعباً ما). (عديلة،

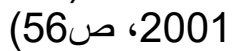

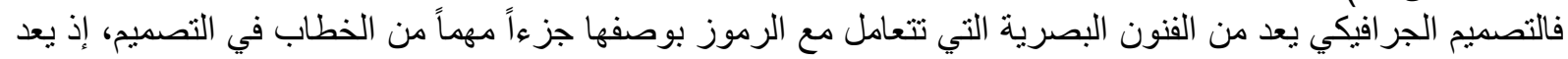

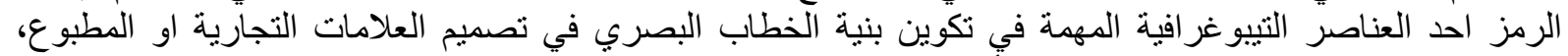

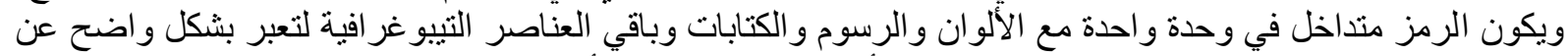

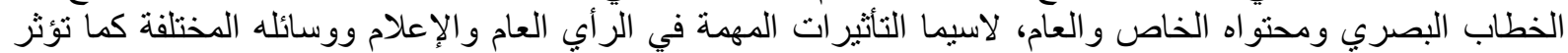

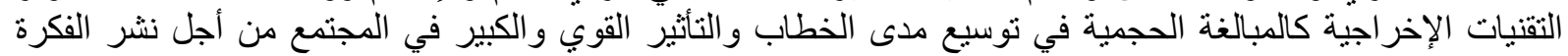

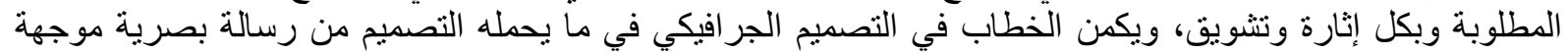

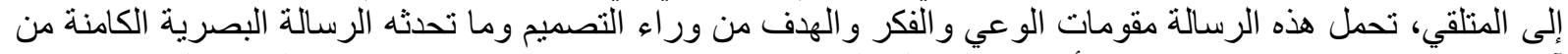

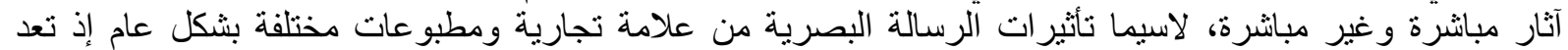

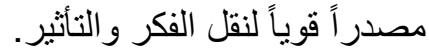

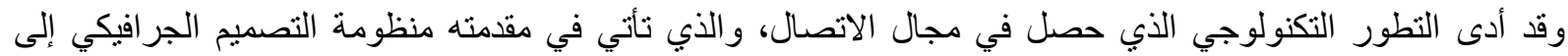

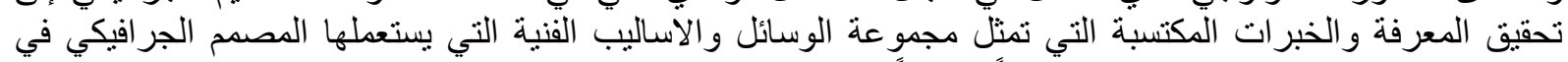

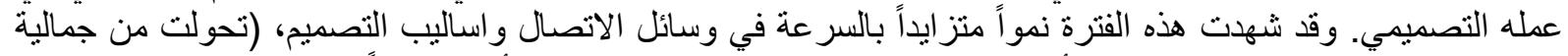

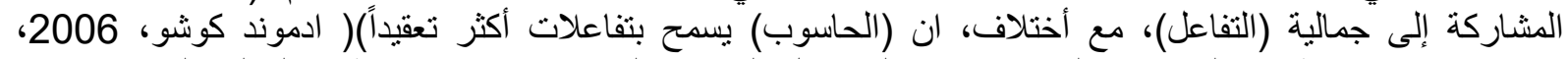

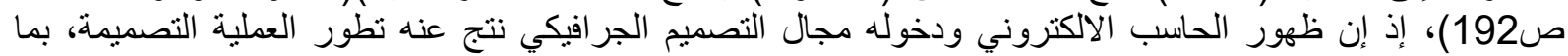


يمتلكه من إمكانات تطويرية وتطبيقية أدت إلى تسارع عمليات الانتاج و إختصار الزمن والكات والكلفة وتحقيق الجانب الجمالي

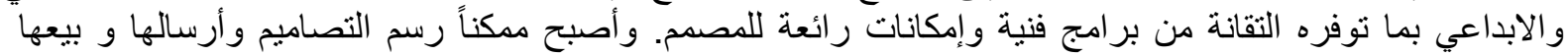

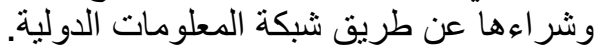

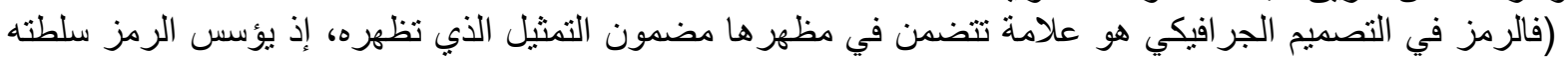

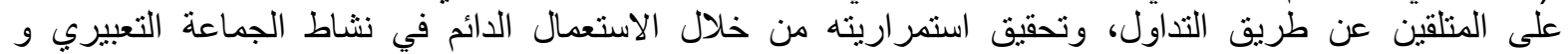

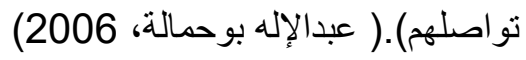

\section{تصميم العلامة التجارية}

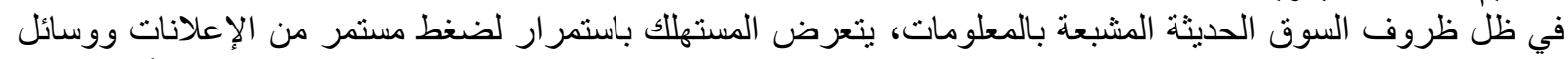

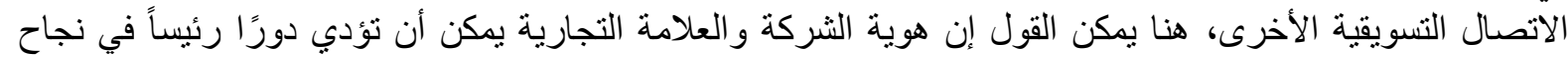

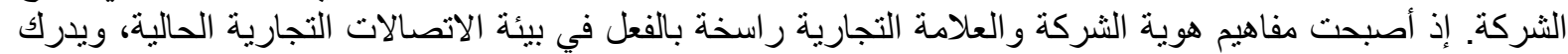

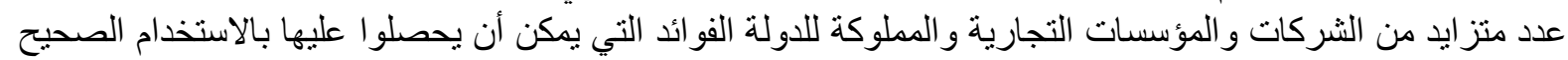

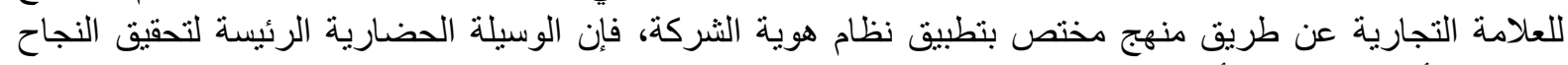

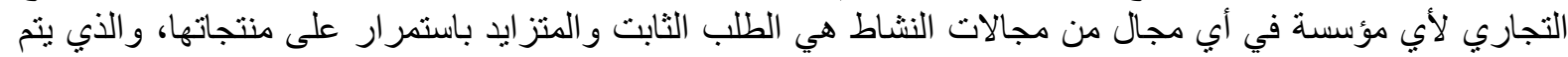

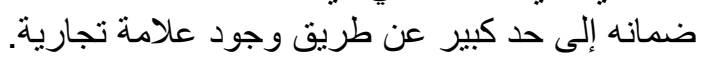

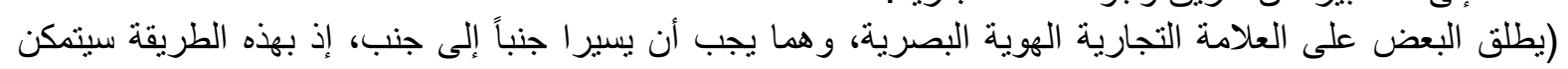

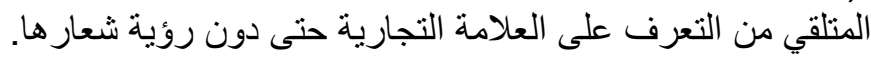

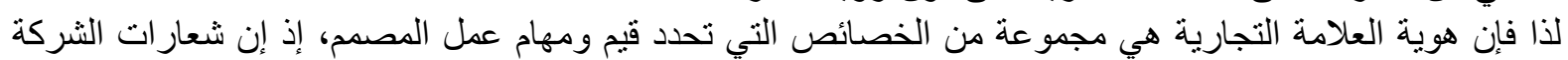

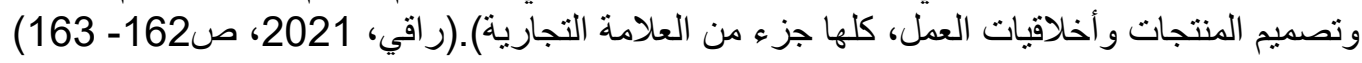

\section{الخصائص الأساس لتصميم العلامة التجارية:}

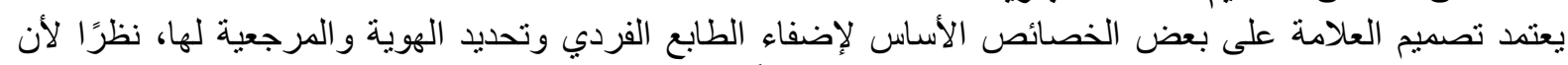

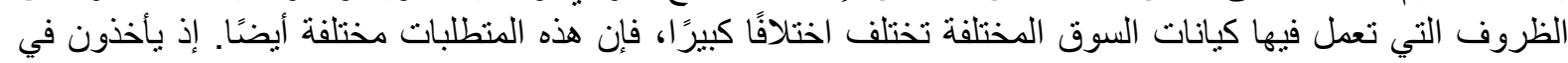

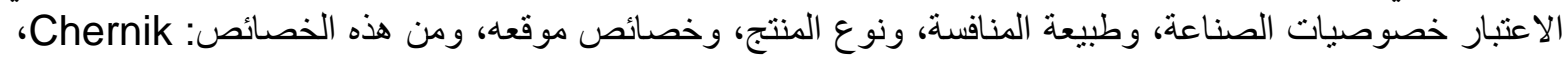
2002. P.18

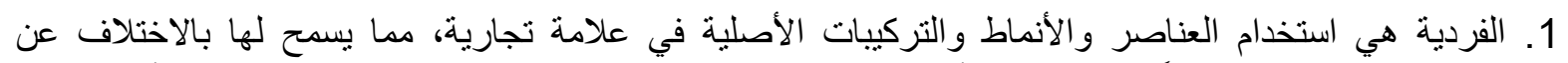

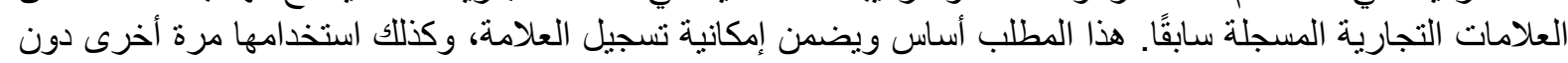

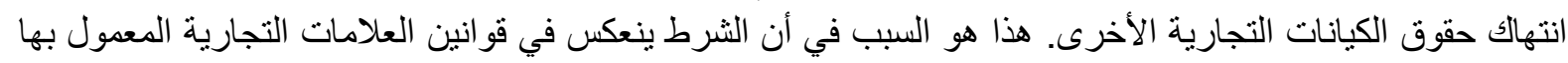
في مختلف البلدان. 2. البساطة هي مطلب استشاري بطبيعته ويتضمن استخدام عناصر في علامة تجارية يسهل التعرف عليها من قبل التبل

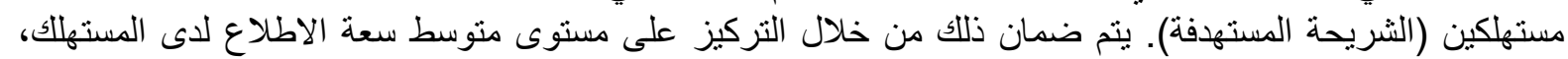

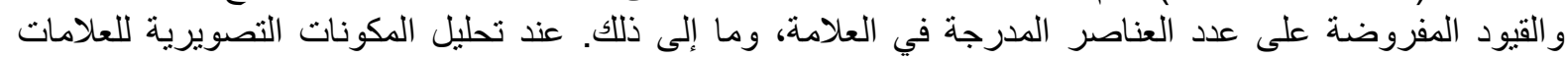

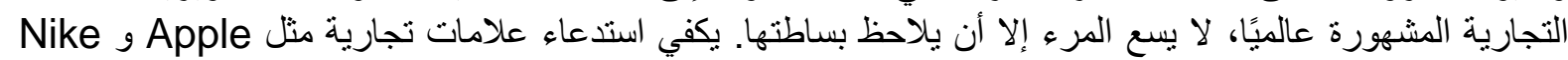
وما إلى ذلك. 3. جاذبية المستهلكين هي ملكية للعلامة التجارية لإثارة المشاعر الإيجابية والجمعيات بين أي مستهلكين مستعملين لهذا

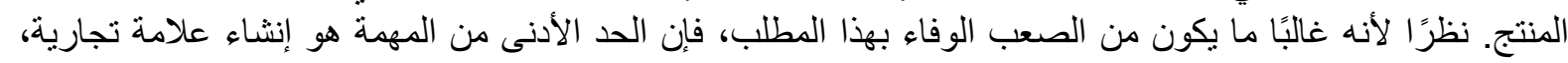

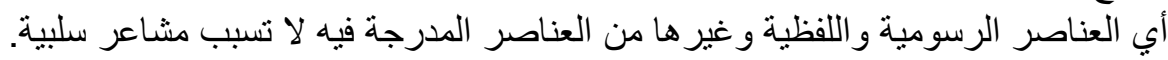

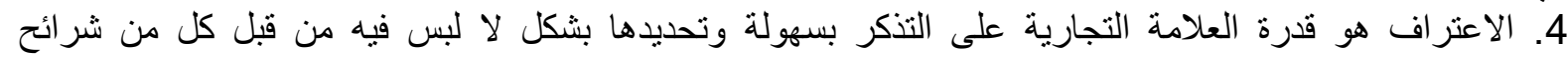

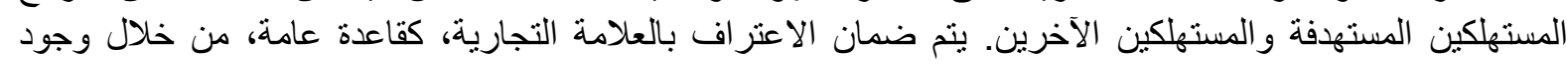
عناصر معبرة وتر ابطية فيها. 5. القابلية للحماية هي قدرة العلامة فئل على التسجيل وفقًا لمنطلبات التشريع، وكذلك الحفاظ على هذه الحالة خلال فترة التسجيل بأكملها.

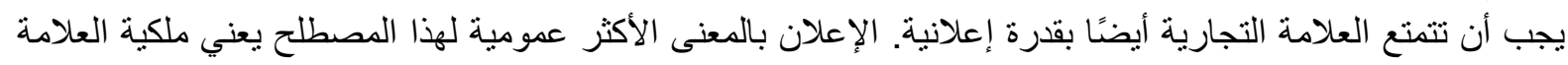

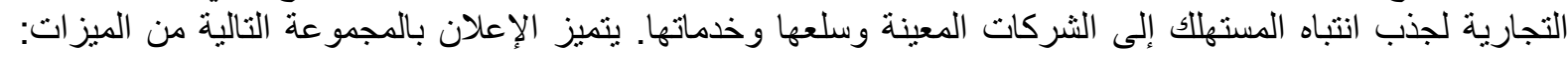
حداثة الفكرة، الجماليات، الإيجاز ، القدرة على التكيف، الترابط، سهولة التهائة النطق، قابلية التصنيع. 
تتضمن قابلية العلامة التجارية للتكيف استدامة العلامة التجارية، أي القدرة على استخدامه لفترة طويلة عند إجراء

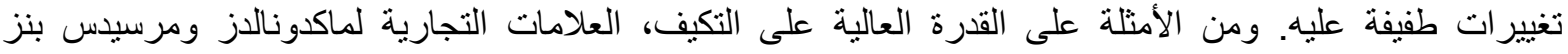

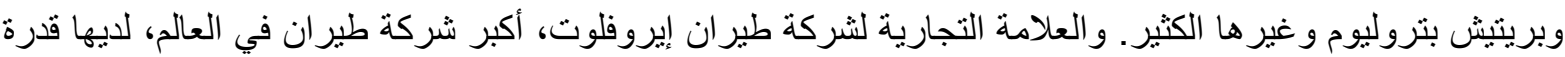

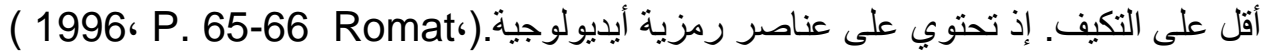

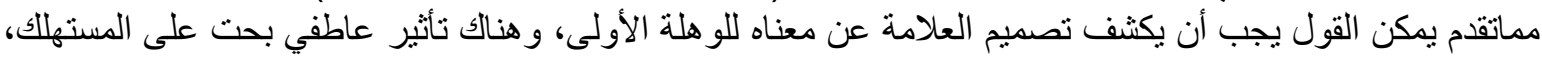

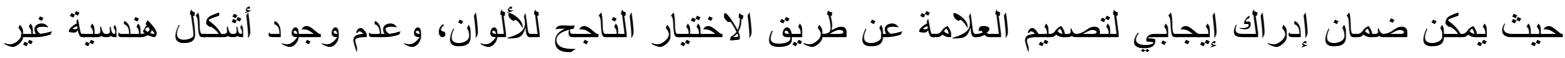

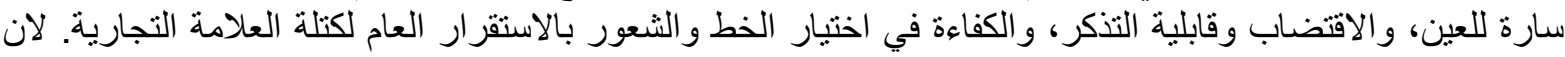

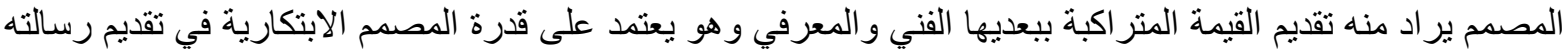

المرئية، ونوجيه أنظار المستهلك نحو الفكرة الأساس في التصمية.

$$
\text { تطبيقات البحث الرابع }
$$

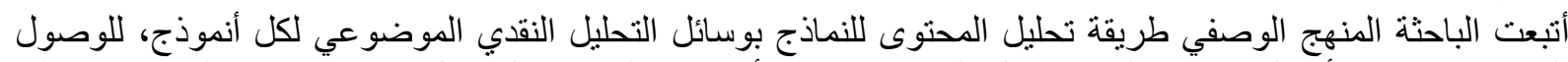

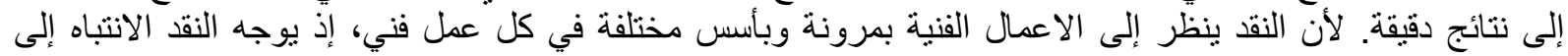

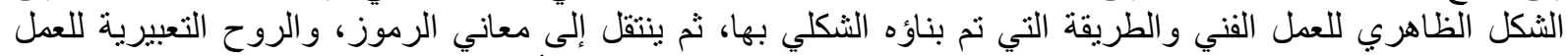

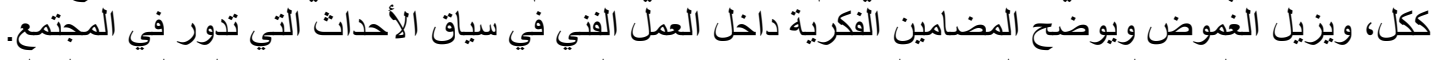

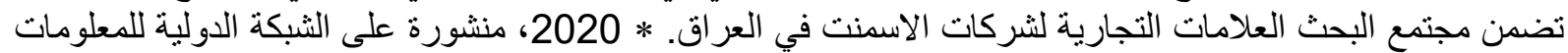

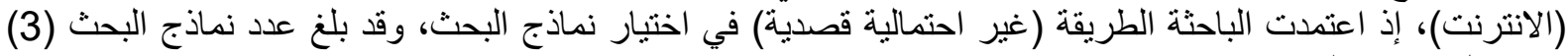

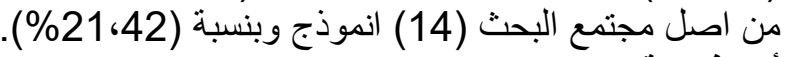

أنموذج رقم (1) - 20

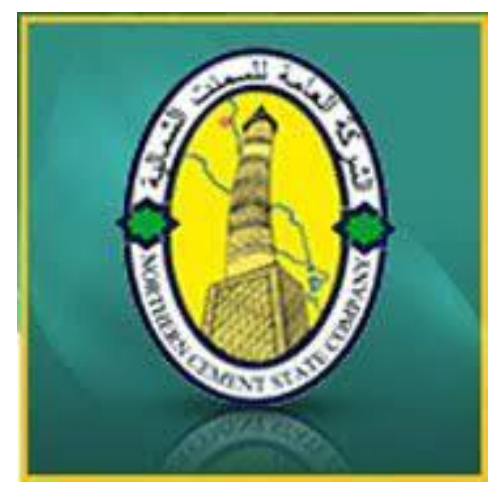

الثركة العامة للسمنت الثمالية في نينوى

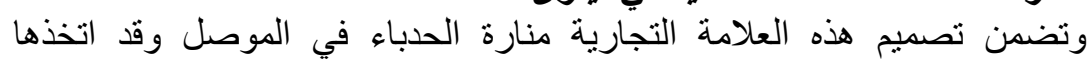

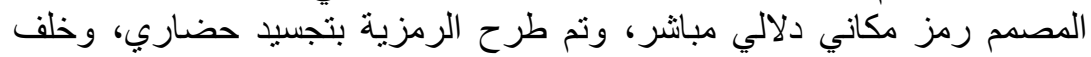

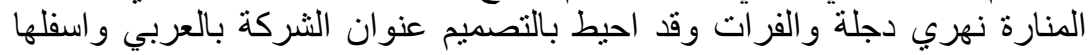

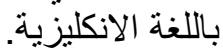

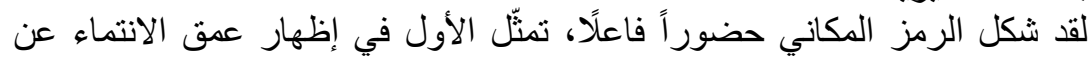

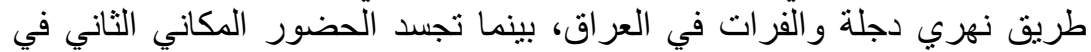

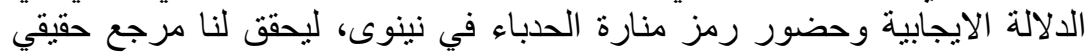

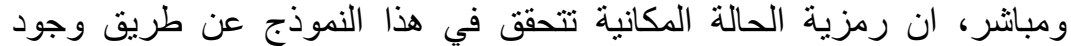
المعاني في البيئة المكانية لتقوية إحساس الافر اد بالانتماء إلى المجتمع و المكان.

أنموذج رقم (2)

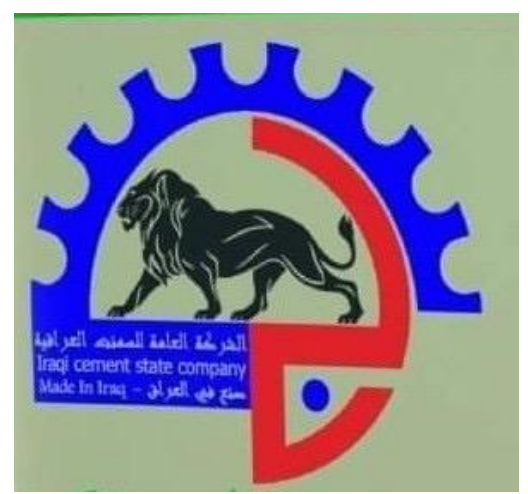


أنموذج (3)

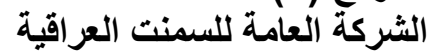

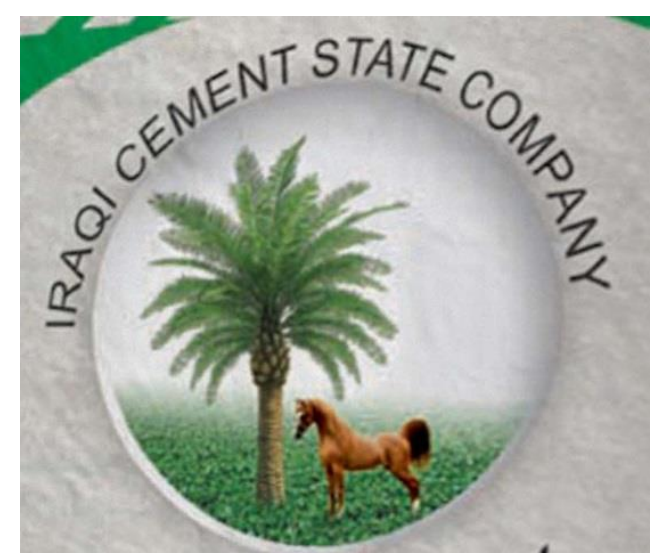

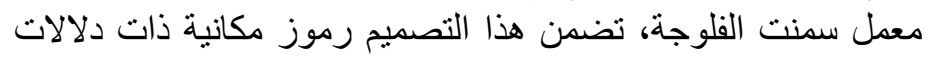

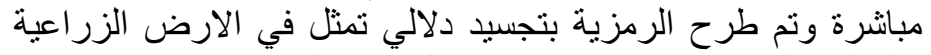

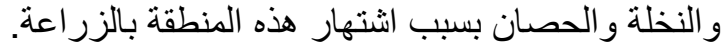

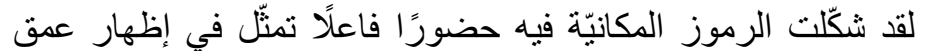

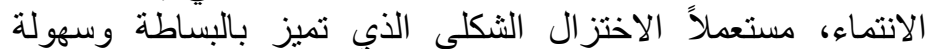

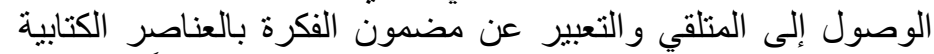

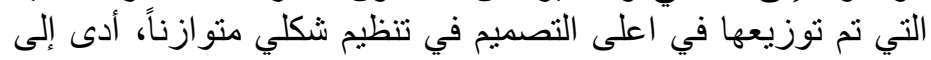

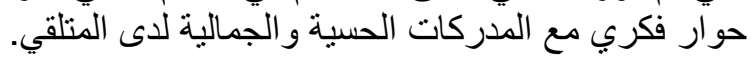

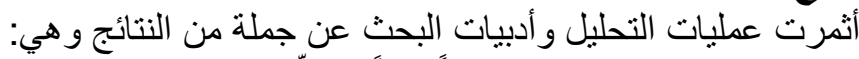
الفصل الثالث نتائج البحث الثرث

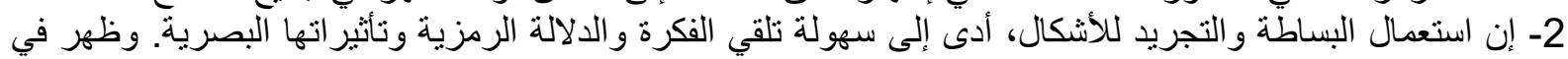

3ـ أديع النماذجمال الاختز ال الثمكلي و الحروفي في تحديد الرمز المكاني بطريقة غير مباشرة وقد ظهر في النموذج (2). استنتاجات البحث

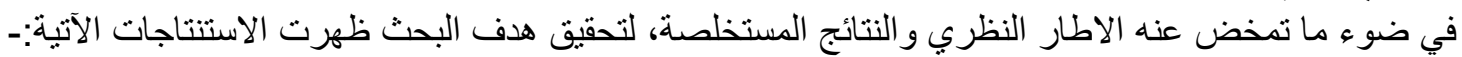

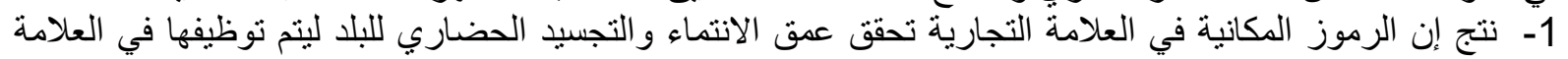

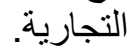
2- حققت الرموز المكانية في تصميم العلامة التجارية تأثير ابداعياً في المتلقي مما أدى إلى التعرف على المرجعيات

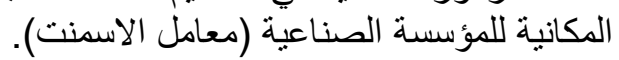

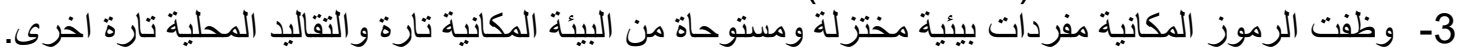

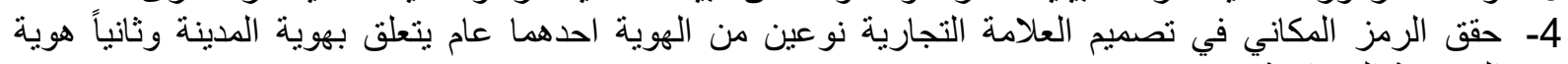
المؤسسة الصناعية.

5- ترتبط رمزية المكان في تصميم العلامة التجارية بتأثير وجود المعاني في البيئة المكانية من خلال احساس الفرد والصورة المعنوية المرتبطة في ذئهنه. توصيات البحث البرة في ضوء

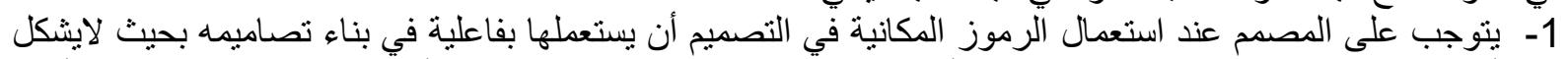

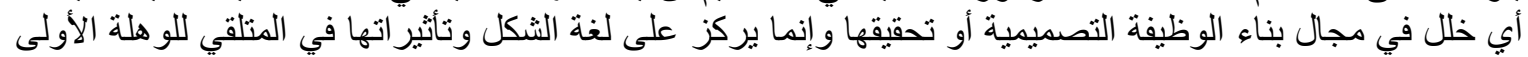

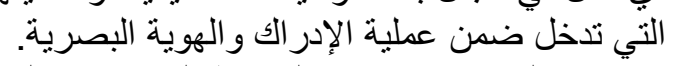

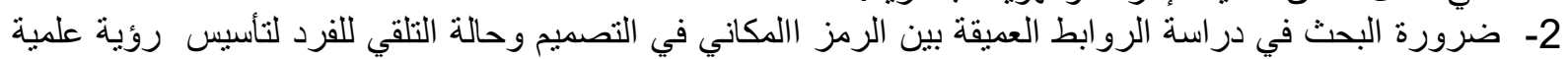

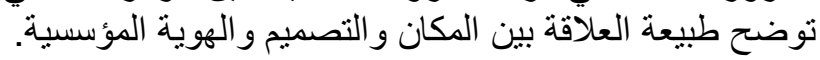

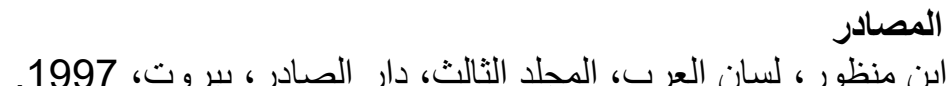

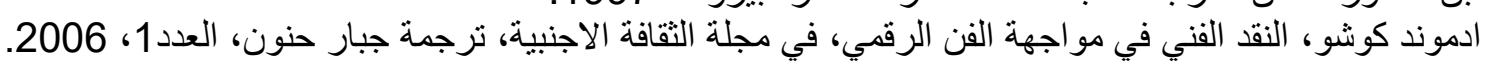

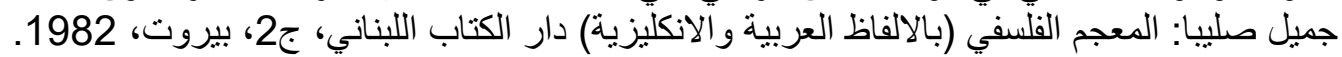

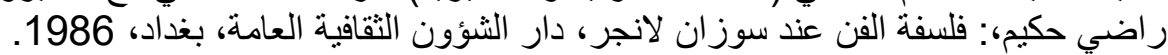

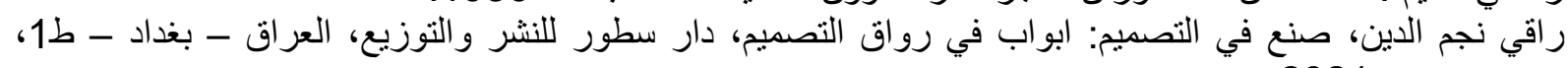
.2021

زغو محمد، أثر العولمة على الهوية الثقافية للأفراد والثعوب، بحث منشور على الثبكة الدولية للأنترنت، الأكاديمية للار اسات الاجتماعية و الانسانية، نيسان، 2010. 
زكريا ابر اهيم: فلسفة الفن في الفكر المعاصر ، مكتبة مصر، 2018.

سلوى محسن حميد، دينا محمد عناد، جماليات تصميم رموز السية السلام في الملصق العالمي المعاصر، دار الذاكرة للنشر

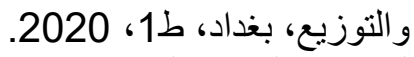

عبدالإله بوحمالة مورفولوجية الرمز و خطاب التميمة، مجلة الحوار المتمدن العدد (1602) 2006/5/ مجلة الكترونية.

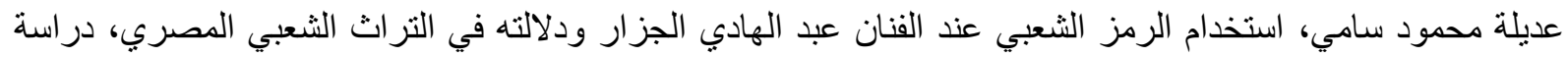

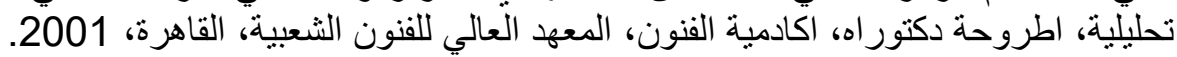

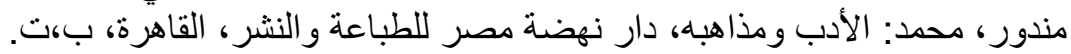

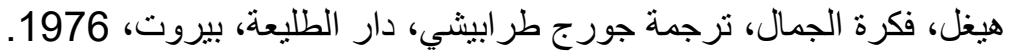

Albert Chavanne، Jean Jacques burst، )، (‘droit de la propriété industrielle، Edition Cinq، dalloz، paris،1998.

Kotler، Ph.، (‘ Marketing Management. 9th Edition )، Prentice Hall Inc، USA. 1997. Lang، John " Creating Architectural Theory" New York، Van Nostrand and Rienhold Company-1987.

Chernik N.Yu. Trademark in the marketing system: Textbook. allowance. Minsk: BSEU، 2002.

Romat E.V. Advertising: Study guide. Kiev، 1996.

https://icsc.gov.iq/ 